\title{
Yan Shigu and The Standardization of Chinese Language
}

\author{
Jinxia Zhang \\ School of Liberal Arts \\ Shandong Normal University \\ Jinan, China \\ e-mail: bobizeus@163.com
}

\begin{abstract}
Yan Shigu, a famous linguist, scribe and historian of the Tang Dynasty, is most famous as a linguist. He attached great importance to Chinese language standardization and had achieved major achievements in this regard through tireless efforts theoretically and practically. His proposal of the combination of the principle of "congzheng" and "suisu" is still enlightening in today's study on language standardization.
\end{abstract}

Keywords-Tang Dynasty; Yan Shigu; Chinese language standardization

\section{INTRODUCTION}

Yan Shigu was a famous linguist, scribe and historian of the Tang Dynasty. According to Tang Shu, originally from Langya (today's Linyi), Yanshigu,whose literary name was Shigu and was called Zhou(someone says the other way around), was born at Jing Zhao Wan Nian (today's Xi'an) in the first year of Sui Kai Huang (581 A.D.). He died at the age of 65 in 645 A.D.. He spent 37 years in the Sui Dynasty and 28 years in the Tang Dynasty, which saw his major accomplishments achieved. Yan Shigu tested and corrected $\mathrm{Wu}$ Jing, decided the final edition of Wu jing Zheng Yi, compiled Zi Yang, noted Han Shu and Ji Jiu Pian and writed Kuang Miu Zheng Su. Today only Han Shu Zhu, Ji Jiu Pian Zhu and Kuang Miu Zheng Su are preserved. Only one edited volume of $\mathrm{Zi}$ Yang exists.

By studying the works of Yan Shigu, we find that Yan Shigu attached great importance to the work of the standardization of Chinese. He had made tireless efforts both theoretically and practically and had achieved significant achievements.

\section{YAN SHIGU'S THEORY ON LANGUAGE SPECIFICATION}

Language (including written language) is the most important instrument of communication for human being. To better play its communicative role, there must be a standard. The so-called standard is that government or individual, by some means, shows members of the society of the same language what is the right way of using that language and what is wrong, so as to popularize the right way and abolish the wrong. Xu Jialu, a famous linguist, once said, 'Standard is a social behavior that appeared not long after mankind entered into civilization, and it's developing in step with social productivity'. Throughout the history, explorations and practices on the standardization of language have never ceased. The Tang Dynasty was a dynasty with powerful comprehensive national strength in both economy and culture. It had a frequent need for foreign exchanges, thus, a strong need for standardized Chinese. Yan Shigu, as a renowned philogist of the early Tang Dynasty who knew the situation he faced and the responsibility he beared clearly, could engage himself in the work to standardize Chinese consciously and had made indelible contributions in this regard.

The overall principal when Yan Shigu standardized Chinese was "follow the ancient rules instead of current fashion". In the prelude of Ji Jiu Pian Zhu, he wrote that "Things cannot be discussed without testing. If we have to discuss, then follow the ancient rules since they are credible." However, he was not an inflexible one that revered the past regardless of the reality. In practice, he could comfort to the reality of language evolution and combine "cong zheng (follow the ancient rules)" and "sui su (follow current fashion)" together, which fit the regularity of language development. Just as Professor Xu Shiyi once said, "In the long term, the evolvement of language is absolute while the stability is relative".

When it comes to Yan Shigu's theory on standard, his grandfather Yan Zhitui certainly could not be ignored for his influence on his grandson in every way. Yan Zhitui was a prominent scholar of the Northern and Southern Dynasty. He paid much attention to education of standardized language to his children. He once said, "I supervise my children on the right way to use language in their childhood; they will be punished whenever they make mistakes, big or small". It is safe to say that the Yan's knowledge on standard Chinese came down in one continuous line.

The Northern and Southern Dynasty was an era of disunity and turbulence, one embodiment in written language was the surge of the amount of Chinese characters and the mess in the use of Chinese characters. Witnessing this picture, Yan Zhitui callED for the unity of typeface between north and south in Chapter Zayi of Yan Shi Jia Xun. But he also believed that characters themselves were changing with the times. Therefore, in Chapter Shuzheng of Yan Shi Jia Xun, he opposed the practice of writing every word in Xiao Zhuan (a style of calligraphy). Due to his realization of the evolvement of language, he advocated that while standardizing language, 
current fashion should not be neglected, that is, to combine "cong zheng" and "sui su" together; otherwise, it may hamper communication. Such attitudes were inherited directly by his grandsons Yan Shigu and Yan Yuansun.Such adaptability are very enlightening in our study today of standard characters and language. "zheng (classic)" and "su(popular)" are essentially relative. With the development of the time and the evolution of language, what's once called "su" can be turned into "zheng". Today, many simplified Chinese characters are the absorption of ancient popular characters.

\section{YAN SHIGU'S PRACTICE ON LANGUAGE STANDARDIZATION}

Having done masses of practices in Chinese standardization, Yan Shigu is surely a representative in standardized language in early Tang Dynasty. Specifically, his work falls mainly on the following areas:

$A$. In the first place, proofread Wu Jing and participate in the edition of Wu Jing Zheng Yi, so as to provide a study model for students at that time to help them take imperial exams.

The Tang Dynasty had inherited the Sui Dynasty culture and education system that set a national exam to employ government officials. But Taizong of the Tang Dynasty thought that some errors had occurred during the circulation of $W u$ Jing and this was detrimental to the employment. Therefore, he asked Yan Shigu to correct Wu Jing. After collecting all sorts of copied $W u$ Jing and its rubbings, referring to wordbooks like Shuo Wen, Zi Lin and Yu Pian, comparing these books and making sure the regular scripts in each book, Yan Shigu finally compiled the Wu Jing Ding Ben. From then on, an official classic book with united characters served as the benchmark for selecting talents.

When issuing $W u$ Jing Ding Ben, as a result of the various schools of Confucianism and of their different teaching methods, Taizong ordered Kong Yingda, director of Guo Zi Jian, Yan Shigu, Sima Caizhang, Wanggong and Wangyan to write hundreds pieces of Wu Jing Yi Xun, named Yi Zan, later renamed Zheng Yi. That is the Wu Jing Zheng Yi in today's Shi San Jing Zhu Shu. Since the publication of the book, all the books that did not agree with Wu Jing Zheng Yi since the Wei Dynasty and the Jin Dynasty were considered heterodoxy, which is of great importance to the unification of Confucianism in the early Tang Dynasty and the imperial examination.

Yan Shigu proofread $\mathrm{Wu}$ Jing and edited Wu Jing Zheng Yi simultaneously. These two projects set a standard for Chinese language by using influential classics as a start. We can see it from the quotation of the final edition in Mao Shi Zheng Yi. When citing the final book, Kong Yingda used it when The Book of Songs had discrepancies. Thus we can see its tremendous role in finalizing classic books and standardizing language.

B. In the second place, write Zi Yang to unify the typeface of regular script.

From the Wei Dynasty and the Jin Dynasty to the Sui Dynasty, China was in a constant state of disunification and wars. Consequently, culture and education development were declining and many mistakes occured in language. The use of language was a mess, some people created words and wrote them casually. Yan Zhitui had his remarks on this situation in Chapter Za Yi of Yan Shi Jia Xun. After the establishment of the Tang Dynasty, Taizong appointed Shigu as Mi Shu Shao Jian, in charge of proofreading documentaries in the seventh year of Zhenguan (633A.D.) to deal with the problems. He could deal with the questions confused by many in rare books and uncommon characters and find the sources. According to the prelude of Gan $\mathrm{Lu} \mathrm{Zi} \mathrm{Shu,} \mathrm{Yan} \mathrm{Shigu} \mathrm{corrected} \mathrm{classic}$ books and wrote them down in papers to show their errors and collate them; the papers were spread among public and thus called Yan Shi Zi Yang, meaning standard character pattern. So, this book, with the purpose of correcting characters, formulated the standard way of writing regular script after considering patterns of the past and present. This book is now extinct. In the republican period of China, Wang Liqing gained one volume of Yan Shi Zi Yang from a sea of books, and embodied the book in the Series of Guang Cang Xue Qiong Cong Shu.

Since the issuance of Ziyang, there appeared a large amount of books like Ziyang, among them the prominent ones included: Qun Shu Xin Ding Zi Yang by Du Yanye, Gan Lu Zi Shu by Yan Yuansun, Wu Jing Wen Zi of Zhangcan, Xin Jia Jiu Jing Zi Yang of Tang Xuandu, to name just a few, with Gan Lu $Z i$ Shu the most popular one. Yan Yuansun, Yan Shigu's brother's grandson, edited Gan $L u Z i$ Shu on the basis of Ziyang. We can therefore know that Yan Shigu's Ziyang must have been widely circulated at that time. And Yan Yuansun must be quite familiar with Ziyang for there are many similarities between Ziyang and Gan Lu Zi Shu. Since Gan Lu Zi Shu are passed down to posterity thanks to Yan Zhenqing's craving, we can catch a glimpse of some characteristics of Ziyang through Gan Lu Zi Shu. Throughout his life, Yan Shigu had many work, including Wu Jing Ding Ben, Ziyang, Kuang Miu Zheng Su, Han Shu Zhu and Ji Jiu Pian Zhu. Many of his theories were interconnected with these books. For instance, his old-age work Kuang Miu Zheng Su and Han Shu Zhu share many similarities. It can be inferred that Ziyang is independent with other books. By comparative study, we find that some of Yan Shigu's analysis of characters scattered in Han Shu Zhu, Ji Jiu Pian Zhu and Ziyang also appeared in Gan Lu Zi Shu. Thus, Gan Lu Zi Shu was an inheritance of Ziyang. We can draw the conclusion that, through the comparison between Gan Lu Zi Shu and Ziyang, the aim of Yan Shifu's writing of Ziyang was to deal with the mess in writing characters since the Wei Dynasty and the Jin Dynasty, set a unified model for characters and correct the right meaning and usage of characters so as to standardize characters.

\section{In the third place, rectify common errors and standardize Chinese language and characters.}

In Kuang Miu Zheng Su, Han Shu Zhu and Ji Jiu Pian Zhu, Yan Shigu always rectified the popular mistakes in the society to standardize Chinese language and characters. The mistakes covered from lexical and phonetics to semantics. Specifically, lexically, he differentiated some homonyms in those books. Phonetically, he pointed out that there was a tendency that people pronounced the words with the same meaning in the 
same way. For example, “chang（尝）” and “zeng (曾)”were of the same meaning, and people would then pronounce "chang" as "zeng". Yan Shigu was opposed to such practices. In his view, although the meanings of these words were alike, their sounds should not be confused. Semantically, for example, he criticized Yingshao's interpretation of "yang

（恙）” of “Wuyang(无恙)” in Feng Su Tong Yi as man-eater bug. He said that there was no such thing as man-eater bug, "wuyang" meant worriless.

\section{CONCLUSION}

In conclusion, language standardization was a great job that Yan Shigu committed to his whole life. Realizing the necessity of language standardization and having a clear objective and feasible approach, he achieved enormous achievements in his work, and will surely stamp his name on the page of history for later generation's extolment.

\section{REFERENCES}

[1] Li Jianguo, A Brief Introduction to the History of Chinese Language Strandardization, Beijing: Language\&Culture Press, 2000.

[2] Liu Xiaodong, Remarks on Kuang Miu Zheng Su, Jinan: Shandong University Publishing House, 1999.

[3] Xu Shiyi, Study on Hui Lin Yi Yin, Shanghai: Shanghai Academy of Social Science Publishing House, 1997 